\title{
Wavelet Transform Analysis of Truncated Fringe Patterns in 3-D Surface Profilometry
}

\author{
Sai Siva Gorthi* and KameswaraRao Lolla \\ Department of Instrumentation, Indian Institute of Science, Bangalore 560012, INDIA
}

\begin{abstract}
Wavelet transform analysis of projected fringe pattern for phase recovery in 3-D shape measurement of objects is investigated. The present communication specifically outlines and evaluates the errors that creep in to the reconstructed profiles when fringe images do not satisfy periodicity. Three specific cases that give raise to non-periodicity of fringe image are simulated and leakage effects caused by each one of them are analyzed with continuous complex Morlet wavelet transform. Same images are analyzed with FFT method to make a comparison of the reconstructed profiles with both methods. Simulation results revealed a significant advantage of wavelet transform profilometry (WTP), that the distortions that arise due to leakage are confined to the locations of discontinuity and do not spread out over the entire projection as in the case of Fourier transform profilometry (FTP).
\end{abstract}

Keywords: wavelet transform, fringe projection, surface profile, FTP, leakage effect, phase demodulation, WTP.

\section{INTRODUCTION}

The automatic measurement of an object shape is very important in industrial quality control, machine vision and solid modeling applications. Several 3-D object profilometry methods including phase measurement profilometry (PMP), Fourier transform profilometry (FTP), modulation measurement profilometry (MMP), spatial phase detection (SPD) etc., have been exhaustively studied. Among these, owing to its ability to evaluate the phase from a single fringe pattern $^{1}$, FTP became the most preferred choice for dynamic applications. Since the introduction of FTP, several improvements have been suggested during last two decades ${ }^{2}$.

The common procedure in extracting the object profile through FTP involves- projecting a sinusoidal fringe pattern on to the object from an offset angle and recording the image of the pattern which is phase modulated by the topographical variations of the object surface. FFT analysis is then carried out to extract the phase from the deformed fringe pattern from which height distribution of the object can be calculated. Although Fourier transform profilometry has grown into a mature technique, some important restrictions still exist in the range of geometries that can be measured. This is inherently tied to the use of a Fourier transform where the fringe and height distributions are assumed to be periodic with the image window, if not distortions (leakage) will result (the effect will be illustrated in Sec-4).

Recently some attempts were made to avoid this leakage and thereby reduce the errors in the reconstructed profiles. A common approach to this problem is using some filters ${ }^{3}$ to reduce the effect, which, however, results in a diminishing frequency resolution. Effectiveness of pre-transform windowing in tackling leakage problem was evaluated ${ }^{4}$. Windowing reduces the magnitude of fringes at the outer edges of the image to such an extent that the phase there is no longer discernable. Moreover, the reduction in errors with the use of pre-transform window is found to be only a few percent if the noise level is more than $\sim 10 \%$. Recently Vanherzeele et al., ${ }^{5}$ have proposed a method that do not exhibit this leakage effect where in, instead of applying a Fourier transform on the full image, the image is divided into small partitions. For each of these partitions phase and frequency of the fringes is estimated using a regressive Fourier transform technique.

*Email: saisiva@isu.iisc.ernet.in; Phone +91-080-22932741; Fax: : +91-080-23600135 
Computational load of this technique is quite heavy, because for every window first of all least squares has to be performed to generate initial values for the maximum likelihood equations which in turn have to be iterated at least about 10-20 times. This process has to be repeated $\left(M-M_{0}\right) * N$ times to evaluate the entire image $(M \times N)$.

On the other hand, very recently only, use of WT analysis in phase recovery from projected fringe pattern for 3D shape measurement was introduced for the first time ${ }^{6}$ and its advantage over Fourier transform with respect to the applicable limit (maximum measurable slope of object surface) was demonstrated ${ }^{7}$. Just as FFT analysis, WT analysis can also extract phase information from a single fringe pattern. Even though few papers mentioned the use of wavelet transform for the extraction of phase of fringes ${ }^{8,9}$, no reference to its effectiveness in handling issues related to leakage problem faced in FTP have been found. This paper evaluates for the first time, errors that creep into the calculation of the three-dimensional shape of an object using wavelet transform analysis from a non-periodic fringe image. Sec-2 provides a brief description of FTP and issues related to leakage. Description of WTP is presented in Sec-3. In Sec-4 different height distributions which cause leakage are simulated and profiled through FTP and WTP methods. Sec-5 lists the conclusions.

\section{FOURIER TRANSFORM PROFILOMETRY (Takeda's Method) ${ }^{1}$}

This section briefly summarizes the procedure involved in analyzing a fringe pattern through FFT method. The intensity profile of the recorded image when fringe pattern is projected on to the object is given by

$$
g(x, y)=a(x, y)+b(x, y) \cos \left(2 \pi f_{0} x+\varphi(x, y)\right)
$$

where $\varphi(x, y)$ contains the information about the object profile and $a(x, y), b(x, y)$ represent unwanted irradiance variations arising from the non-uniform light reflection by a test object. This equation can be rewritten as

$g(x, y)=a(x, y)+c(x, y) \exp \left(j 2 \pi f_{0} x\right)+c^{*}(x, y) \exp \left(-j 2 \pi f_{0} x\right)$ where $c(x, y)=1 / 2 b(x, y) \exp (j \varphi(x, y))$

* denotes complex conjugate. Next above Eq. is Fourier transformed with respect to $\mathrm{x}$ by the use of a fast Fourier transform algorithm, which gives

$$
G(f, y)=A(x, y)+C\left(f-f_{0}, y\right)+C *\left(f-f_{0}, y\right)
$$

where capital letters denote the Fourier spectrum and $\mathrm{f}$ is the spatial frequency in the $\mathrm{x}$ direction. Since the spatial variations of $\mathrm{a}(\mathrm{x}, \mathrm{y}), \mathrm{b}(\mathrm{x}, \mathrm{y})$ and $\varphi(\mathrm{x}, \mathrm{y})$ are slow compared with the spatial frequency $\mathrm{f}_{0}$, the Fourier spectra in above equation are separated by the carrier frequency $\mathrm{f}_{0}$. One can make use of either of the two spectra centered around the carrier, say $C\left(f-f_{0}, y\right)$ and translates it by $f_{0}$ on the frequency axis towards the origin to obtain $C(f, y)$. The unwanted background variations $\mathrm{a}(\mathrm{x}, \mathrm{y})$ has been filtered out in this stage. Again using inverse FFT, inverse Fourier transform of $\mathrm{C}$ (f, y) w.r.to $\mathrm{f}$ is computed to obtain c (x, y).

The phase part of $\mathrm{c}(\mathrm{x}, \mathrm{y})$ can be separated from the rest by calculating the imaginary part of the complex logarithm:

$$
\varphi(\mathrm{x}, \mathrm{y})=\operatorname{Im}\{\log [\mathrm{c}(\mathrm{x}, \mathrm{y})]\}
$$

The phase map for the whole image is formed by repeating this process for each horizontal line in the image. However the phase thus obtained is indeterminate to a factor of $2 \pi$ (wrapped phase). The phase unwrapping algorithm can correct these discontinuities.

Although different authors have shown independently that the Fourier transform method works well on various examples, there is an important restriction on the applicability: ${ }^{10}$

The image $\mathrm{i}(\mathrm{x}, \mathrm{y})$ should be periodic (values on the left and right side of the image borders should be equal). If this is not the case leakage will occur (the energy of a spectral line at frequency $\mathrm{k}$ will spread out over its neighboring frequency lines).

This means that in order to avoid distortions in the reconstructed image with classical Fourier transform methods a few 'rules' have to be respected. 
$>$ The carrier frequency $\mathrm{f}_{0}$ should be chosen in such a way that the image $\mathrm{i}(\mathrm{x}, \mathrm{y})$ contains an integer number of fringes.

$>$ The height distribution $\mathrm{h}(\mathrm{x}, \mathrm{y})$ itself should be periodic in the time window (this is for example satisfied when the object under investigation falls completely within the image because then the height is zero near the border of the image).

The origin for the leakage can be seen by considering the following simple one dimensional sinusoidal signal:

$$
\mathrm{i}(\mathrm{x})=1 / \mathrm{M} \Sigma \mathrm{I}(\mathrm{k}) \exp 2 \pi \mathrm{i}(\mathrm{k} / \mathrm{M}),
$$

where I(k) are the FFT coefficients. The exponential basis functions that are used in the approximations have the frequencies $\mathrm{f}_{\mathrm{k}}=\mathrm{k} / \mathrm{M}$ for $\mathrm{k}=1, \ldots, \mathrm{M} / 2$. If the spatial carrier frequency $\mathrm{f}_{0}$ of the original sinusoidal signal $\mathrm{i}(\mathrm{x})$ is not equal to one of FFT frequencies $\mathrm{f}_{\mathrm{k}}$ (e.g., $\mathrm{f}_{0}=1.5 / \mathrm{M}$ ), leakage will occur (see Fig. 1).

In this paper analysis of images which do not satisfy the periodicity condition is carried out with both Fourier transform method and Wavelet transform method and the results are compared. Next section briefly summarizes procedure involved in wavelet transform profilometry (WTP). For elaborate description reader is advised to go through Ref. 7.

\section{WAVELET TRANSFORM PROFILOMETRY (WTP)}

Wavelets offer a powerful method to quantify how energy is spatially distributed at multiple frequencies. The one dimensional wavelet transform of the fringe signal is defined by

$$
W(a, b)=1 / \sqrt{ } a \int h *[(x-b) / a] g(x, y) d x
$$

Each wavelet is obtained by scaling a mother wavelet $\mathrm{h}(\mathrm{x})$ by a $>0$ and translating it by $\mathrm{b}$. The choice of an appropriate wavelet for a given application is an important practical question. In this study, the Morlet wavelet was used to deal with phase recovery because it is known to provide a better localization in both spatial and frequency domains ${ }^{6}$. The Morlet wavelet, which is a plane wave modulated by a Gaussian, is defined as

$$
\mathrm{h}(\mathrm{x})=\pi^{1 / 4} \exp (\mathrm{icx}) \exp \left(-\mathrm{x}^{2} / 2\right)
$$

where $\mathrm{c}$ is a fixed spatial frequency. Computing the 1-D complex Morlet wavelet transform of the signal only in the $\mathrm{x}$ direction with y fixed, we obtain the phase of WT coefficients by taking the inverse tangent of the quotient of the imaginary part and by dividing it by the real part:

$$
\Phi(\mathrm{a}, \mathrm{b})=\arctan \{\operatorname{imag}[\mathrm{W}(\mathrm{a}, \mathrm{b})] / \operatorname{real}[\mathrm{W}(\mathrm{a}, \mathrm{b})]\}
$$

and the amplitudes of WT coefficients are defined as

$$
\mathrm{A}(\mathrm{a}, \mathrm{b})=\left(\{\operatorname{imag}[\mathrm{W}(\mathrm{a}, \mathrm{b})]\}^{2}+\{\operatorname{real}[\mathrm{W}(\mathrm{a}, \mathrm{b})]\}^{2}\right)^{1 / 2}
$$

The desired phase distribution $\varphi(x, y)$ which contains the information about the object topographical variations can be obtained from the phase $\Phi(\mathrm{a}, \mathrm{b})$ at ridge points ${ }^{7}$ (The maximum of the WT amplitudes at every position is defined as the ridge of the WT coefficient). Phase distribution of the entire image can be obtained by repeating this process for each value of $y$. It should be noted that the phase function obtained is a wrapped phase map and unwrapping is required to get continuous profile.

Because the instantaneous frequencies $f_{k}$ (or equivalently scales) that can be used in the WT analysis is arbitrary, leakage can be reduced. (i.e., $\mathrm{f}_{\mathrm{k}}$ is not limited to the frequencies of the FFT frequency lines). WT analysis can be carried out at different range of scales with variable resolution and thus the unknown local frequency can be estimated with better accuracy (see Fig. 5.6). 


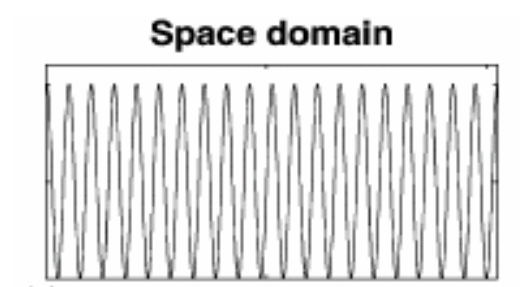

(a)

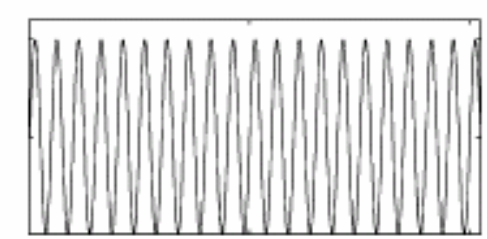

(b)
Frequency domain
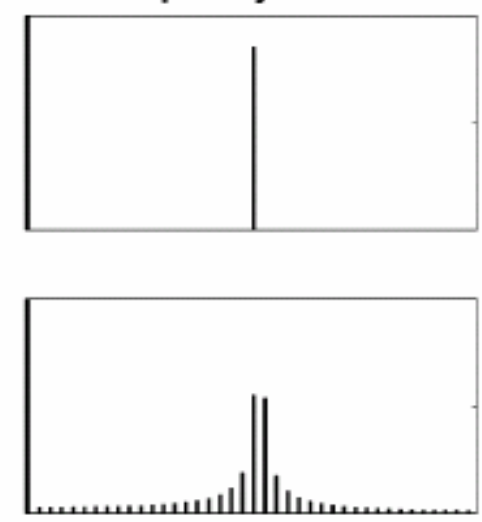

Figure. 1 (a) sine wave with integer number of periods and its spectrum (b) non-integer number of periods

\section{COMPUTER SIMULATIONS AND DISCUSSIONS}

To evaluate the performance of WTP in handling non-periodic fringe images, an object with the following height distribution is simulated and processed. When any one of the following condition is satisfied then the image becomes non-periodic. The same images are analyzed with FTP and reconstructed profiles are compared against the true profile. The height distribution of the object considered for simulation is same as the one illustrated in Ref. 10. The simulated object has the height distribution given by:

$$
h(x, y)=\cos \left(2 f_{x} x\right) * \sin \left(2 f_{y} y\right)
$$

This height distribution allows simulations of varying distortions which can occur in practice (Fig.2).

- A carrier frequency $\mathrm{f}_{0}$ which does not coincide with a frequency line of the FFT grid, which simply means that the number of fringes in the image is not an integer.

- The height distribution $\mathrm{h}(\mathrm{x}, \mathrm{y})$ is not periodic in the time window (i.e. $\mathrm{f} \mathrm{x}$ or $\mathrm{f} \mathrm{y}$ are not integers).

- A discontinuity in the height distribution.

Fig. 3 shows the simulated image of the projected fringe pattern with non integer number of fringes on the object with periodic height distribution. Frequency spectrum of this image (along a row) is shown in Fig. 4, which clearly shows the presence of leakage. As can be seen, when a considerable amount of energy goes out of the filter band width, nonnegligible errors creep into the reconstructed 3-D profile. The presence of higher harmonics makes the process even more difficult. Fig 7(a) shows the reconstructed profile with 2D-FFT method. In our work wavelet transform analysis is carried out with MATLAB. Graphical User Interface wavelet tool box enables to analyze the signals just by setting various parameters in the window such as range of scales, resolution, sampling period and type of mother wavelet etc. Fig 5 show the image of the complex continuous 1-D wavelet transform GUI menu. As is already pointed out earlier, accuracy of measurement depends on the accuracy with which instantaneous local frequency of the pattern is estimated. This in turn depends on the resolution of the scale used in calculating wavelet coefficients. Thus first WT is calculated over a large range of scales with less resolution. Then ridges (corresponding scale values at each position where the WT coefficients have maximum amplitude) are identified. Then again WT is computed over very small range of scales centered on ridge points with increased resolution. This procedure ensures reduction in leakage by accurately estimating instantaneous frequency. This is illustrated in Fig. 5,6. 


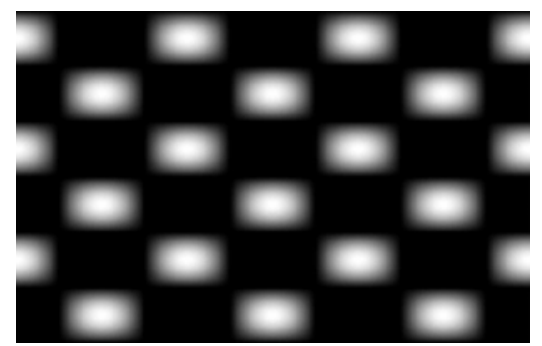

Fig.2 Simulated height distribution $\mathrm{h}(\mathrm{x}, \mathrm{y})$

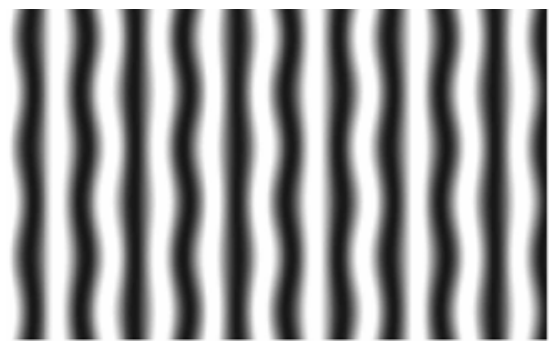

Fig.3 Intensity image of projected fringes with carrier frequency $\mathrm{f}_{0}=10.5$

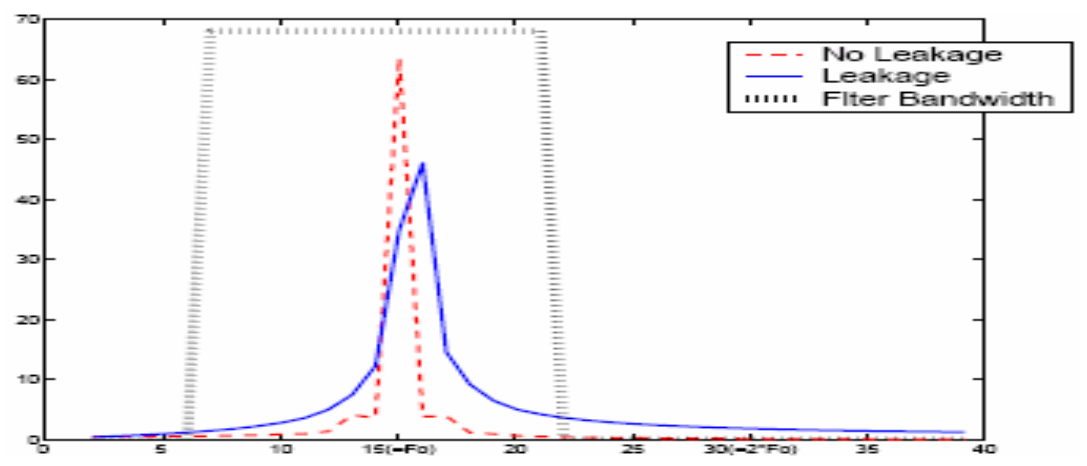

Fig. 4 Frequency spectrum of image in Fig.2 along a row (exhibiting leakage)
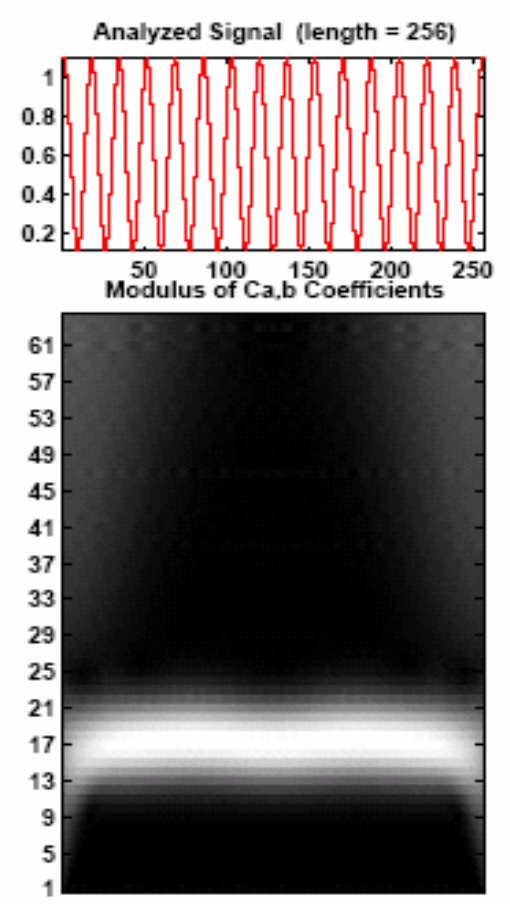

Scale of colors from MIN to MAX
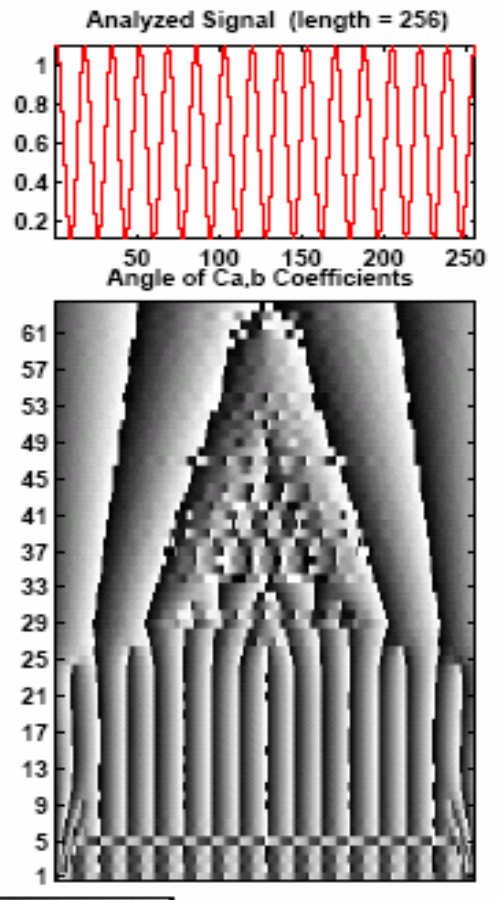

$\mathrm{X}-\mathrm{Y}$

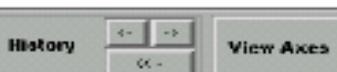

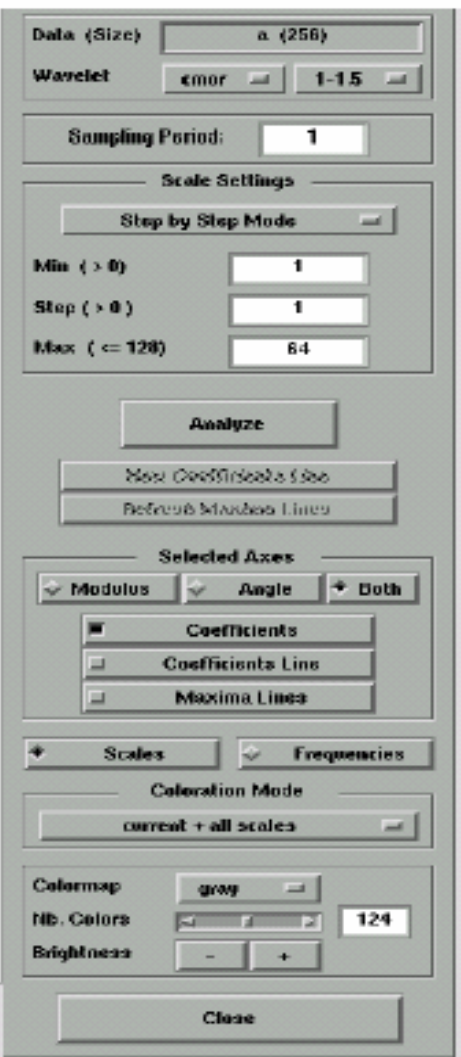

Fig.5 Image showing GUI tool box in MATLAB for Wavelet analysis 

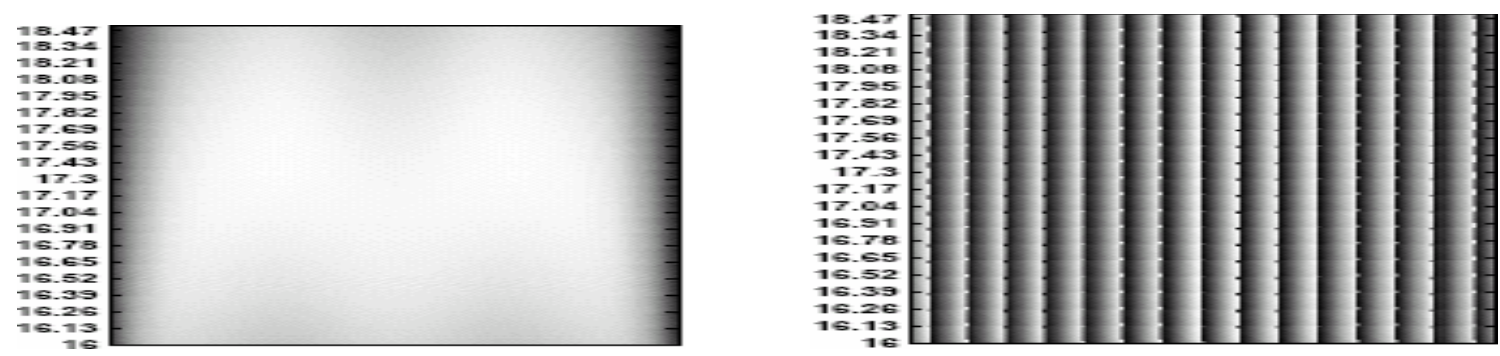

\section{Scale of colors from MIN to MAX}

Fig. 6 Amplitude and phase of wavelet coefficients calculated with increased resolution of scale near ridges (position (x or b) along $\mathrm{x}$-axis and scale/dilation factor (a) along $\mathrm{y}$-axis)
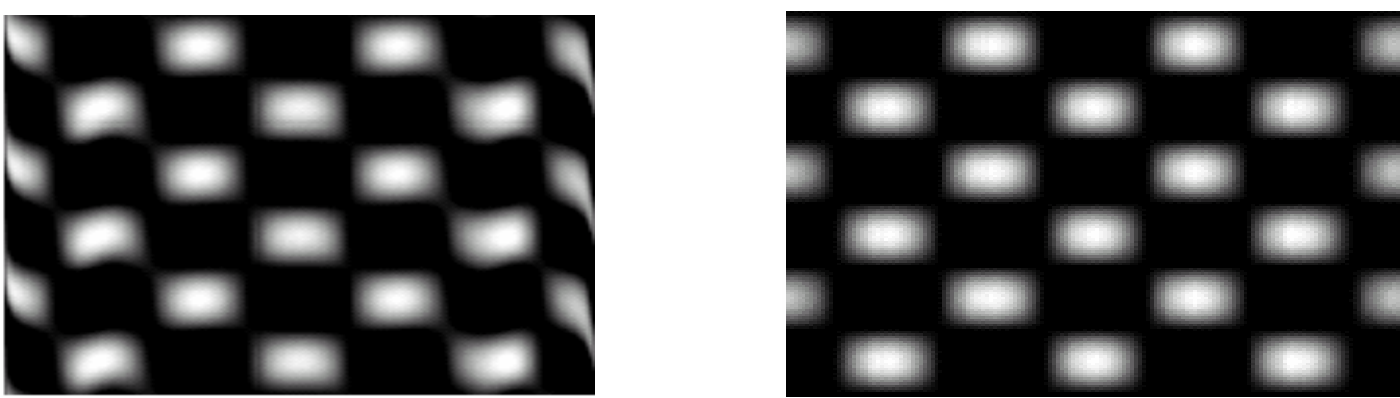

Fig.7 Height demodulated from Fig. 3 using (a) 2D-Fourier transform and (b) Wavelet Transform analysis
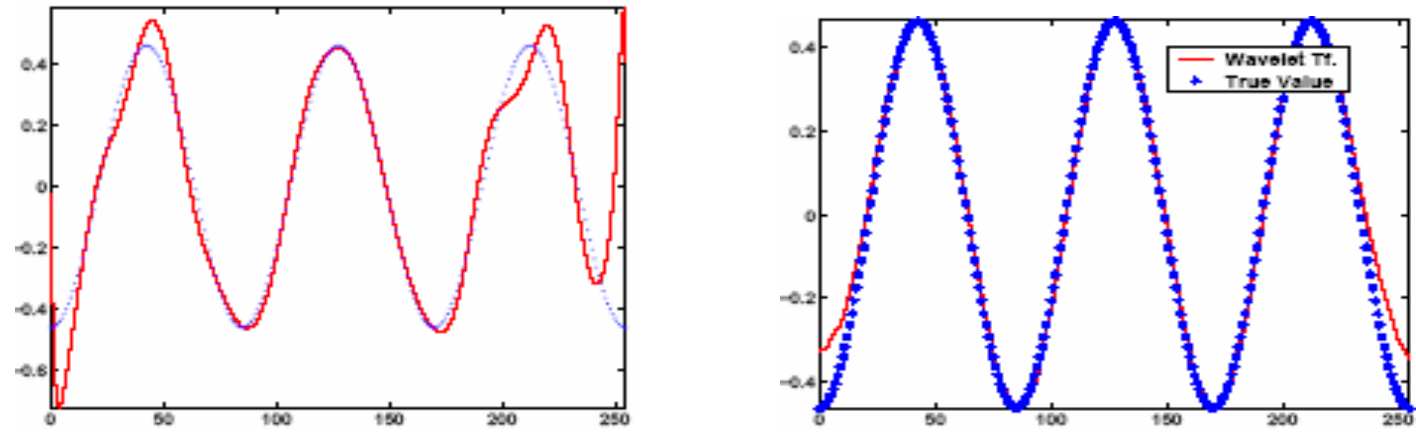

Fig. 8 Height demodulated from Fig. 3 along $135^{\text {th }}$ row using (a) Fourier transform and (b) Wavelet Transform analysis

As it was already pointed out in ${ }^{10}$, leakage due to a non-periodic height distribution is of even greater importance. The most important reason is a practical one. When measuring large objects one usually has to relay on taking separate sub images. This almost guarantees that the height will not be the same at the borders, which means a discontinuity and in turn leads to leakage. On top of that these distortions are not confined to the image edges but are spread out over the entire projection. Fig. 9 show image of simulated non-periodic height distribution and Fig. 10 shows the results obtained by FFT and WT analysis. Fig. 11 shows the comparison of reconstructed profiles along a row with true profiles. To see the effect of surface discontinuity on the reconstruction of profile, the height distribution shown in Fig. 12 is simulated. Fig. 13 shows amplitude and phase of WT coefficients as Gray scale images. As can be seen from the Fig. 14, 15, WT analysis gives very good results except for the region near the step of the image. It is very clear that even though at locations of discontinuities there is small error in reconstruction it does not propagate and affect other regions in the WT method. 


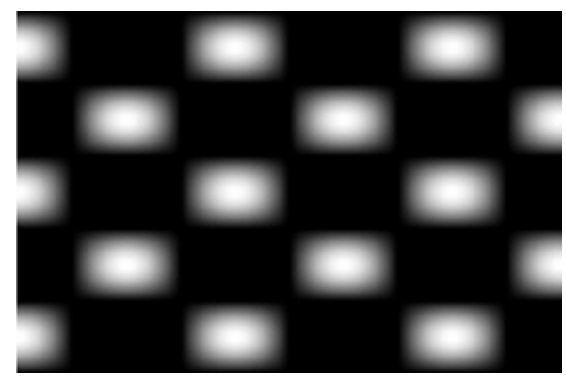

Fig. 9(a) Height distribution with $\mathrm{f}_{\mathrm{x}}=2.5, \mathrm{f}_{\mathrm{y}}=2.5$ (Non-periodic distribution)
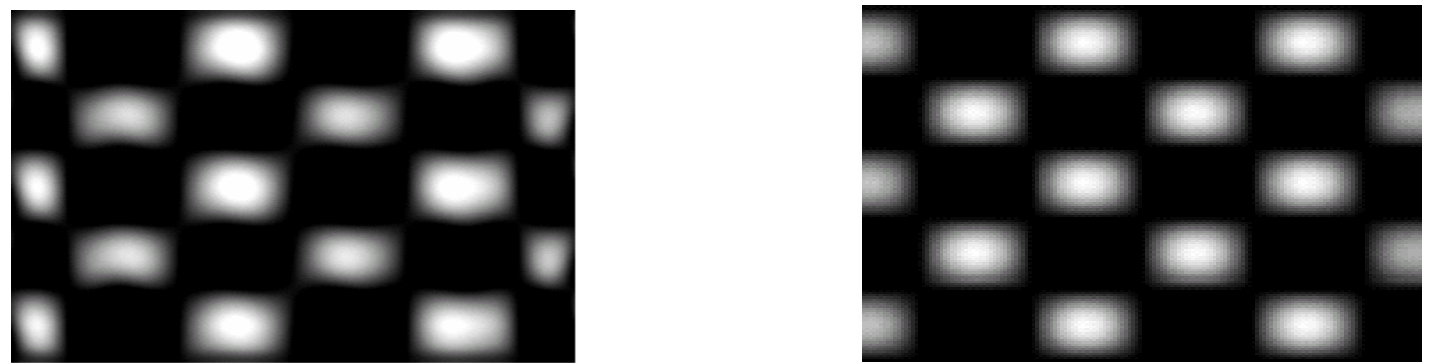

Fig.10 Height demodulated from Fig. 9(b) using (a) Fourier transform and (b) Wavelet Transform analysis
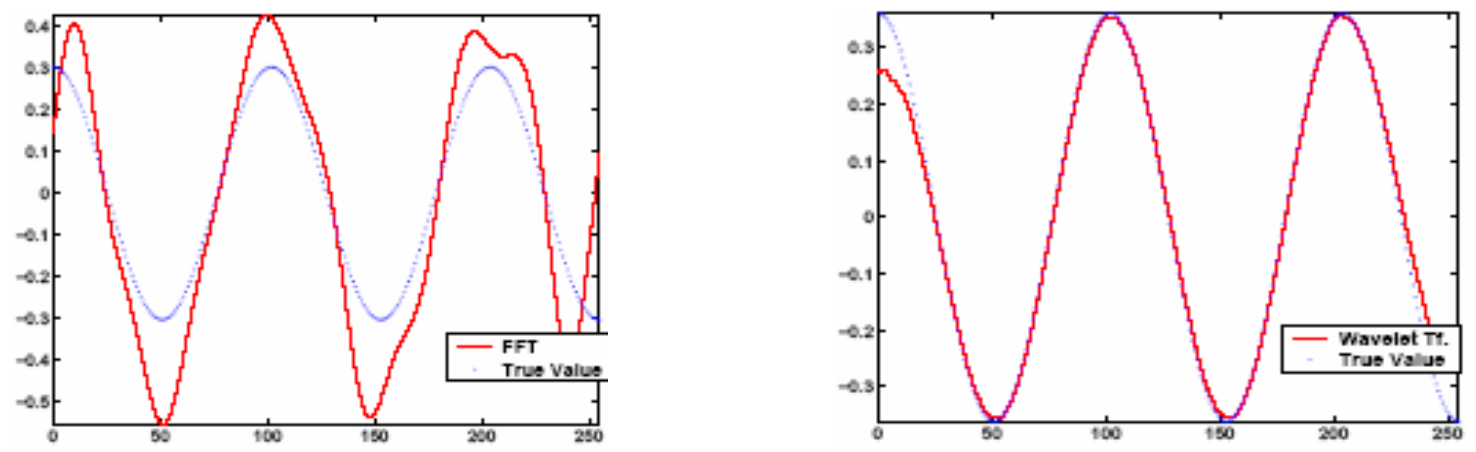

Fig.11Height demodulated from Fig. 9(b) along $250^{\text {th }}$ row using (a) Fourier transform and (b) Wavelet Transform analysis

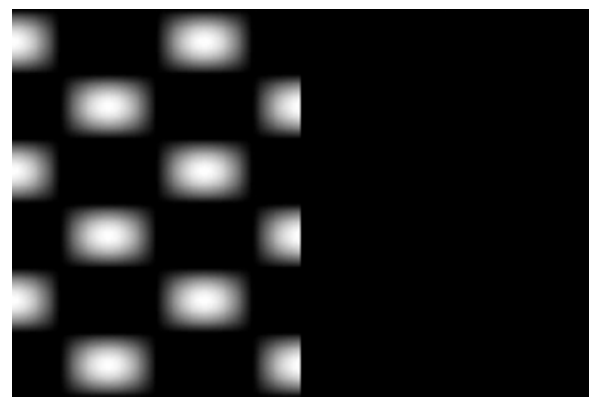

Fig.12 (a) Height distribution with a step

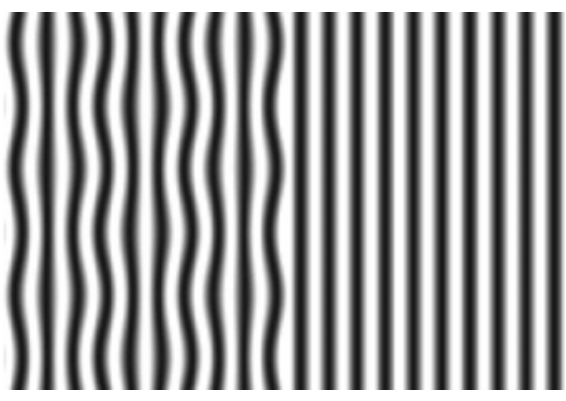

(b) Intensity of projected fringes 

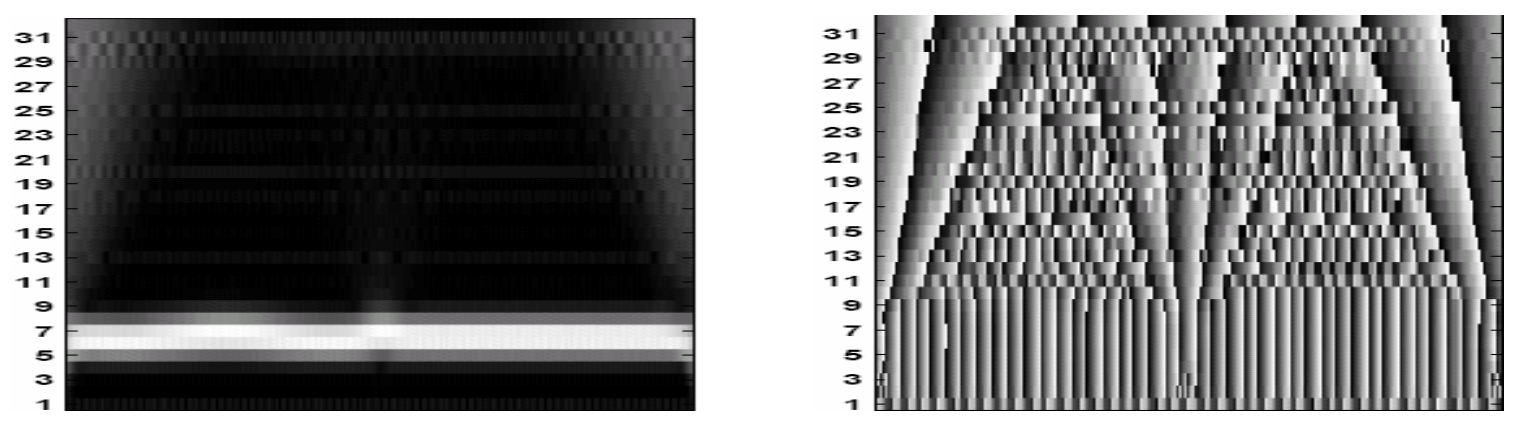

\section{Scale of colors from MIN to MAX}

Fig. 13 Amplitude and phase of wavelet coefficients represented as gray scale map (position/pixels (x or b) along $\mathrm{x}$-axis and scale/dilation factor (a) along y-axis)
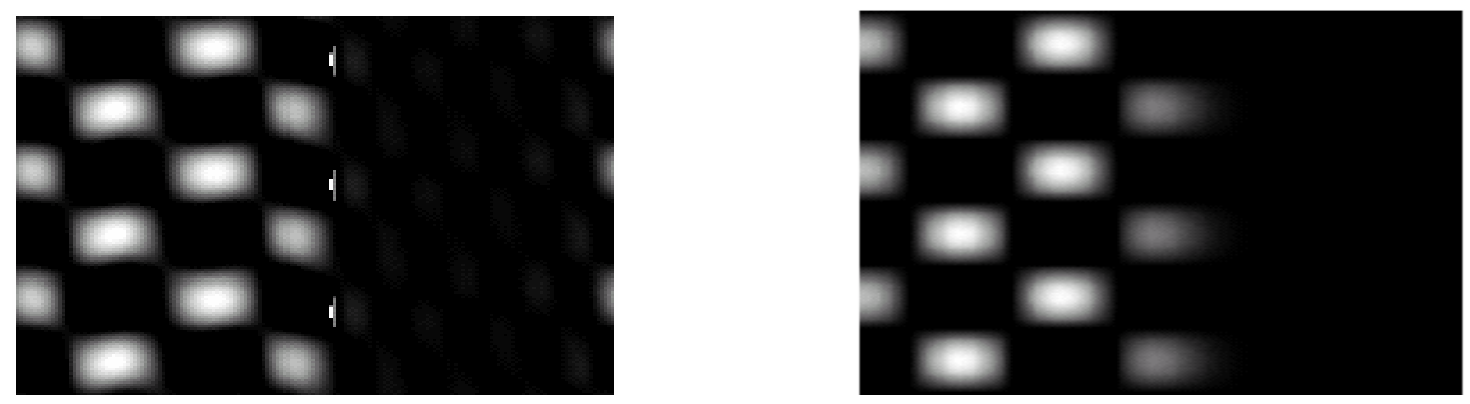

Fig.14 Height demodulated from Fig. 12(b) using (a) Fourier transform and (b) Wavelet Transform analysis
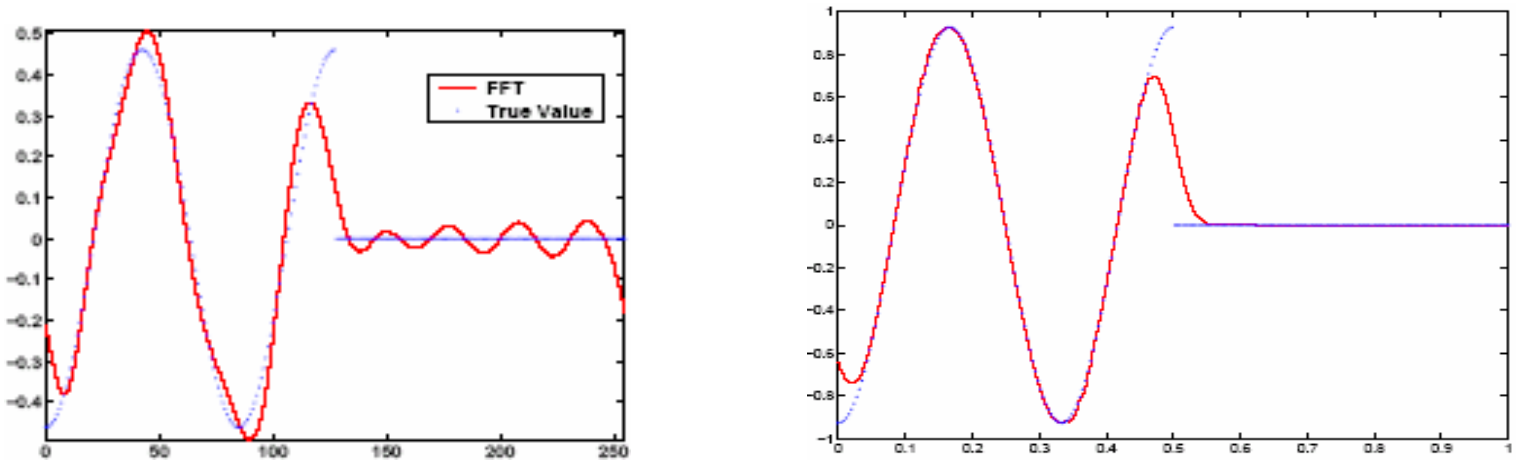

Fig.15. Height demodulated from Fig. 12(b) along $135^{\text {th }}$ using (a) Fourier transform and (b) Wavelet Transform analysis 


\section{CONCLUSIONS}

Performance of wavelet transform profilometry in handling non-periodic fringe images is evaluated. By varying either geometry of the object surface or the illumination conditions three typical cases which introduces non-periodicity in fringe image are simulated and analyzed with WTP. To verify its ability in handling issues related to leakage, same objects are profiled with FFT method and errors in the reconstructed profiles obtained from both methods are compared. It is observed that the performance of WTP is superior to that of FTP. The significant advantage of using WTP is that the errors in the reconstruction are confined to the locations of discontinuities and does not spread out over the entire projection. As it was already demonstrated in literature that other important problems faced in the use of FTP such as maximum measurable slope of the object and robustness against noise are well handled with WTP, and also as our work shows some important advantages of it in issues related to leakage, wavelet transform profilometry can become a reliable and promising tool for 3-D shape measurement in future.

\section{ACKNOWLEDGMENTS}

The authors wish to acknowledge Yogesh C Diwan for his useful suggestions at various stages of this work.

\section{REFERENCES}

1. M. Takeda, K. Mouth, "Fourier transform profilometry for the automatic measurement of 3-D object shapes," Appl Opt 22 (24), pp. 3977-82, 1983.

2. X Su, W Chen, "Fourier transform profilometry: a review," Opt Laser Eng. 35, pp. 263-84, 2001.

3. J Lin, X Su, "Two dimensional Fourier transform profilometry for the automatic measurement of threedimensional object shapes," Opt Eng 11, pp. 3297-302, 1995.

4. F. Berryman, P. Pynsent, J. Cubillo, "The effect of windowing in Fourier transform profilometry applied to noisy images," Opt and Laser Eng. 41 pp. 815-825, 2004.

5. J. Vanherzeele, P. Guillaume, S. Vanlanduit, "Fourier fringe processing using a regressive Fourier-transform technique," Opt and Laser Eng. 43 (6), pp. 645-658, 2005.

6. A. Dursun, S. Ozder and F. N. Ecevit, "Continuous wavelet transform analysis of projected fringe patterns," Meas. Sci. Technol. 15 pp. 1768-1772, 2004

7. J. Zhong and J. Weng, "Spatial carrier-fringe pattern analysis by means of wavelet transform: wavelet transform profilometry," Appl Opt 43(26) pp. 4993-4998, 2004.

8. L. R. Watkins, S. M. Tan and T. H. Barnes, "Determination of interferometer phase distributions by use of wavelets," Opt Letters 24 (13), pp. 905-907, 1999.

9. M. Afifi, A. Fassi-Fihri, M. Marjane, K. Nassim, M. Siski, S. Rachafi, "Paul wavelet-based algorithm for optical phase distribution evaluation," Opt Commun. 211, pp. 47-51, 2002.

10. S. Vanlanduit, J. Vanherzeele, P. Guillaume, B. Cauberghe, P. Verboven, "Fourier fringe processing by use of an interpolated Fourier-transform technique," Appl Opt 43(27) pp. 5206-5213, 2004. 